\title{
Generación M... Diseñando la cartografía del futuro
}

\author{
Generation M... Designing the cartography of the future
}

\author{
Ana Patricia Pinnel \\ pinnelanapatricia@gmail.com \\ ORCID: 0000-0003-2996-3778 \\ Fundación Educación y Trabajo \\ Buenos Aires - Argentina
}

Roberto Enrique Vola Luhrs

volaluhrs@gmail.com

ORCID: 0000-0003-3787-3094

Fundación Educación y Trabajo

Buenos Aires - Argentina

Artículo recibido en diciembre 2020 / Arbitrado en febrero 2021 / Aceptado en febrero 2021 / Publicado en mayo 2021

\footnotetext{
RESUMEN | La investigación tuvo como objetivo determinar si los modos de comportamiento en el mundo laboral están condicionados por el hecho de pertenecer a una generación, o si es mayor el impacto de tener que adaptarse a un nuevo orden mundial; mediante un estudio de tipo mixto, corte transversal que, según la etapa, tuvo alcances descriptivo y relacional. Se aplicó las técnicas de la entrevista a profundidad y el censo a una población heterogénea conformada por 188 estudiantes de postgrado de una universidad pública de Buenos Aires, Argentina.

Los resultados demuestran que el hecho de pertenecer a una generación demográfica no determina las conductas, sino que las actitudes y valores son los que las determinan. Se concluyó que la actitud es un factor determinante para adaptarse a las nuevas tecnologías y retos laborales. Asimismo, se propuso un nuevo enfoque de paradigma no encontrado en la bibliografía.

Palabras clave: $\quad$ Trabajo; Desempeño laboral; Tecnología; Generacional; Conocimiento

ABSTRACT $\quad$ The objective of the research was to determine if the modes of behavior in the world of work are conditioned by the fact of belonging to a generation, or if the impact of having to adapt to a new world order is greater; by means of a mixed type of study, crosssectional that, according to the stage, had descriptive and relational scope. In-depth interview and census techniques were applied to a heterogeneous population made up of 188 graduate students from a public university in Buenos Aires, Argentina.

The results show that the fact of belonging to a demographic generation does not determine behaviors, but rather attitudes and values determine them. It was concluded that attitude is a determining factor in adapting to new technologies and job challenges. Likewise, a new paradigm approach not found in the literature was proposed.

Keywords: Job; Job performance; Technology; Generational; Knowledge
} 
INTRODUCCIÓN

La mentalidad "digital" y "colectiva" está impactando en la manera en que las personas perciben el mundo del trabajo y en la repercusión que éste tiene en sus vidas. Mientras que muchos se sienten amenazados pensando que pueden quedar fuera del Sistema, otros en cambio, se convierten en pioneros porque aprenden / aprehenden para adaptarse rápidamente a las nuevas exigencias. "Se ha iniciado un proceso de mutación $y$ metamorfosis, casi imperceptible, sin embargo, ya lleva años en el medio, habiendo tenido un impacto exponencial a partir del año 2020 por el efecto global de la pandemia. Claramente, implica una disrupción, así como una aceleración digital" (Pinnel \& Vola-luhrs, 2020, p. $1)$.

Estos cambios en la sociedad están en la mismísima "médula ósea" de sus miembros. "...ocurren mutaciones que sólo pueden ser heredadas cuando afectan a las células reproductivas" (Gagneten et al., 2008, p. 1).

Las empresas sólo pueden crecer $y$ desarrollarse si sus miembros también crecen y se desarrollan como individuos íntegros, consustanciados con lo que hacen $y$ comprometidos con su propio futuro y el de la organización. Las organizaciones son entidades reales y no sólo una entelequia, como se las ha querido presentar, como si sólo existiesen en la imaginación de sus colaboradores. Las empresas tienen vida propia y tienen una influencia determinante sobre el comportamiento humano de sus grupos de interés y viceversa.

Las generaciones han sido categorizadas como: Baby Boomers, personas que nacieron durante el Baby Boom (natalidad explosiva), que sucedió en algunos países anglosajones en el período posterior a la Segunda Guerra Mundial, entre los años 40 y mediados de los 60 (Corica \& Dinerstein 2009); " $X$ ", constituida por los nacidos a finales de los 60 y los 70, el éxito para ellos es ser jóvenes y lograr el dinero suficiente para hacer lo que los Baby Boomers piensan hacer al final de su carrera laboral (Uviz D'Agostino, 2008); " $Y$ ", conocida también como generación Millennials, es la cohorte demográfica que sigue a la generación $X$ y precede a la generación $Z_{i}$ "Zeta", son nativos digitales, no conciben la posibilidad de un mundo sin conexión ni respuestas inmediatas al alcance de un clic (Espiritusanto, 2016). Existen otras categorías y clasificaciones que no son objeto de este estudio.

El objetivo de la investigación ha sido determinar si los modos de comportamiento en el mundo laboral están condicionados por el hecho de pertenecer a una generación etaria (Baby Boomers, X, Y y Zeta), o si es mayor el impacto de tener que adaptarse a un nuevo orden mundial (globalización, tecnología, disrupciones, virtualidad, redes sociales, contexto VICA, etc.), impone conductas más allá de la generación a la que uno pertenece.

Para alcanzar el objetivo, se desarrollaron las siguientes tareas: primero, se determinó el grado de dificultad para producir un cambio de programación mental (mindset) que permita al individuo adaptarse al entorno; en segundo lugar, se estableció en qué grado se podría observar, en las distintas generaciones, la resistencia al cambio y si esta resistencia era/es "patrimonio" de alguna en particular, como lo manifiesta el prejuicio social generalizado; finalmente, se encontró denominadores comunes que permitan trascender $o$ no al estereotipo generacional.

La justificación estratégica del estudio, en lo que a organizaciones y a individuos se refiere, 
está en que ellos podrán saber el porqué de algunas de sus conductas clave y pensamientos que determinan su hacer, pudiendo de esta manera superar cualquier etiquetamiento social. Nótese que, "las investigaciones más modernas sobre las pautas de comportamiento de la gente, en los ambientes laborales, han podido establecer que todas las personas tienen potencialidad para trabajar en equipo" (VolaLuhrs, 2007, p. 27).

\section{METODOLOGÍA}

Se trató de un estudio de tipo mixto (cuantitativo y cualitativo) de corte transversal que, según la etapa, tuvo alcances descriptivo y relacional. Consistió en analizar las distintas generaciones en los ámbitos laborales, la relación de éstas entre sí y el modo en que las organizaciones actúan con paradigmas que obstaculizan o desarrollan su propio crecimiento y el potencial de su gente.

Para el cometido se aplicó el método inductivo-deductivo, que permitió, a partir de la teoría existente, cuestionar la conducta de las organizaciones y de las personas en el ámbito laboral. Las preguntas que surgieron son las siguientes: ¿Qué comparten y qué no comparten estos grupos generacionales cuando están en un mismo trabajo?, ¿cuáles son los valores que exige el contexto y por qué son esos y no otros?, ¿cuáles son los desvalores que impactan en la cotidianidad de sus vidas, más allá del tiempo dedicado a sus labores?, ¿por qué resulta indispensable que conozcamos las diferencias por sobre todas las similitudes?, ¿cuáles son las motivaciones más profundas?, ¿son las motivaciones un correlato de la edad?

Para responder a estas interrogantes se aplicó un censo que estuvo dirigido a estudiantes de maestrías en administración de empresas de la Facultad Regional General Pacheco de la Universidad Tecnológica Nacional UTN FRGP (Buenos Aires - Argentina), que cursaron este programa académico en el periodo 2016-2020. Se escogió este universo poblacional debido a que está conformado por profesionales en distintas áreas del conocimiento, que desempeñan funciones en distintos sectores y desde diferentes niveles jerárquicos. Asimismo, en términos generacionales se cuenta con representantes de los cuatro grupos de interés (Baby Boomers, $X, Y$ y Zeta). El único criterio de exclusión fue: participantes que no contaban con experiencia laboral al momento de aplicar el instrumento.

El universo poblacional estuvo conformado por 188 estudiantes de postgrado, de los cuales $165(87,77 \%)$ respondieron la boleta censal y 23 (12,23\%) no pudieron hacerlo por diferentes motivos (abandono, ausencia por licencia laboral o enfermedad).

Como lo muestra la Tabla 1, la población estudiada estuvo conformada por las cuatro generaciones de interés del estudio. 


\section{Tabla 1}

Distribución de la población según año en el que fue consultada y la generación a la que pertenece.

\begin{tabular}{cccccc}
\hline Año & BB & $\mathbf{X}$ & $\mathbf{Y}$ & $\mathbf{Z}$ & TOTALES \\
\hline 2016 & 15 & 11 & 6 & 0 & 32 \\
2017 & 10 & 12 & 8 & 5 & 35 \\
2018 & 7 & 9 & 11 & 6 & 33 \\
2019 & 4 & 8 & 10 & 11 & 33 \\
2020 & 1 & 5 & 12 & 14 & 32 \\
\hline TOTALES & $\mathbf{3 7}$ & $\mathbf{4 5}$ & $\mathbf{4 7}$ & $\mathbf{3 6}$ & $\mathbf{1 6 5}$ \\
\hline
\end{tabular}

La relación de años presentada en la Tabla 1 , corresponde a los 5 años en los que se recogieron los datos (cada estudiante fue consultado una sola vez por más que hubiera estado matriculado durante más de una gestión). La primera fila de la tabla presenta las generaciones: $\mathrm{BB}=$ generación Baby Boomers, 1949-1968; $X=$ generación $X, 1969-1980 ; Y=$ generación Millenials, 1981-1993; y $Z=$ generación Zeta, 1994-2010 (Concejo Edurne, 2018).

En términos generales, es decir, sin distinción del año de recolección de datos, la población estuvo distribuida de la siguiente manera: $B B=29 \%, X=27 \%, Y=22 \%, Z=22 \%$. La presentación de resultados y la interpretación de estos no consideró la distinción del año de recolección de la información, puesto que sólo hacen referencia a la técnica de recogida de datos, pero no corresponden a subgrupos de interés para el análisis.

La boleta censal incluyó 6 preguntas destinadas a recoger la percepción de la población respecto a los motivos determinantes para su éxito en la inserción a una organización y al mercado laboral en general. Todas las preguntas (dimensiones) admirieron 3 opciones de respuesta: Actitud, Generación Biológica (referida a la clasificación demográfica en cohortes de grupos etarios), y Conocimiento (know-how). Siendo la respuesta el factor determinante desde la perspectiva del estudiante.

Una vez interpretados los resultados, según los \% asignados por cada generación a cada una de estas 3 opciones, los investigadores agruparon los resultados en respuestas dicotómicas, según se detalla: Cero = Ausencia = NO Reconocimiento del Estereotipo de la generación como factor determinante $(+)$ para su éxito en la inserción a una organización / al mercado en el contexto VUCA (acrónimo inglés compuesto por cuatro términos: volatilidad, incertidumbre, complejidad y ambigüedad) disruptivo actual; Uno $=$ Presencia $=$ Reconocimiento del Estereotipo de generación como factor determinante $(+)$ de su éxito en la inserción a una organización / al mercado en el contexto VUCA disruptivo actual.

El instrumento cuenta con validación interna, dado que fue sometido a criterio de expertos en Psicología Cognitiva, Neurociencia, Coaching Ontológico y Comunicación. 
RESULTADOS

Como resultado de la aplicación de la técnica de la entrevista, se pudo comprender que, efectivamente, existen estereotipos, a saber:

Miembros de la generación Baby Boomers manifestaron que entre sus preocupaciones está la dificultad para gestionar su tiempo y dinero. Por un lado, sus padres viven más tiempo y, por otros, sus hijos han "estirado" su adolescencia o están en busca de una mejor preparación universitaria, lo que ha retrasado su ingreso al mundo del trabajo. Se trata de una generación menos optimista, con gran desconfianza en los gobiernos por la sobrada corrupción o inoperancia, sin embargo, han asumido un importante compromiso por lo social.

Ellos se han tenido que "adaptar" al uso la tecnología estando ya en sus puestos de trabajo, y lo han logrado. Añoran las reglas claras y simples: "el espacio del trabajo debería limitarse a la oficina y las responsabilidades deberían ser individuales y muy específicas", manifiestan. Sienten un gran respeto por las jerarquías. Se sienten exitosos cuando pueden demostrar que han logrado estabilidad económica y laboral, se han mantenido en una profesión abrazada ya a temprana edad. Suelen argumentar que los logros son el resultado de la permanencia, la persistencia y los sacrificios; la recompensa llegará cuando alcancen el reconocimiento tanto como el bienestar económico en el retiro, y/o la jubilación.

En relación con la generación $X$, se encontró que ésta redefinió la relación entre el trabajo y el tiempo de la recompensa. Estas personas se muestran muy seguras, competitivas y hacen todo lo posible por tener un crecimiento rápido. Buscan mayores ingresos a través de nuevas propuestas profesionales-laborales. De hecho, cuando se analizan sus desarrollos de carrera se observa que, un porcentaje menor son los que han permanecido trabajando para su primer empleador. El éxito, para los " $\mathrm{X}$ ", es ganar dinero, tener poder $y$ liderazgo, ser independientes profesionalmente. Valoran los posgrados como mecanismo para alcanzar sus metas más rápido. Han aceptado desbalancear su vida entre el trabajo y el ocio con alta resiliencia, a cambio de lograr una posición económica sólida rápidamente. La jornada laboral fue extendida al Happy Hour mezclándose de esta manera la vida personal con la profesional. La generación $\mathrm{X}$ se convirtió, así, en adicta al trabajo.

Según las conversaciones con la generación $Y$ (Millennials), se entiende que estas personas necesitan constante retroalimentación para sentir que sus esfuerzos están siendo reconocidos, su nivel de resiliencia es bajo porque en general no han desarrollado la capacidad de sobrellevar la frustración. La oferta que hacen las organizaciones, de crecimiento en la pirámide de carrera laboral, no los atrae en el largo plazo. Muestran entusiasmo en los ambientes de trabajo sustentados sobre la base de la igualdad, solidaridad y respeto mutuo. Cuando de tareas se trata, mostraron especial interés por descubrir, innovar y probar una parte esencial de ellas. Es natural para ellos la diversidad e inclusión. Sostienen que, al conocimiento, que está en ebullición constante, hay que abordarlo tanto de manera formal como informal y que el prueba-error debería ser una normalidad. De hecho, aún en el ámbito del aula, suelen buscar o corroborar el conocimiento en internet más que recurrir a sus docentes. Podrían no respetar la jerarquía y la autoridad, que suelen todavía primar en los modelos organizacionales de las corporaciones. 
Todo su entorno es en tiempo real, "micro momentos". Estar siempre conectados es su ley. Toman decisiones rápidas y exigen inmediatez en las respuestas y en los resultados. Respecto al presente y futuro mostraron, generalmente, una despreocupación por el control. Vivir a pleno el presente es la consigna.

Sí, les preocupa no tener un balance entre su vida personal y el trabajo. Respecto a la vida espiritual y equilibrio emocional, hicieron notar que le dan importancia a la emocionalidad y detestan la insensibilidad de las organizaciones. Valoran el diálogo de igual a igual, lo ven como el mejor reconocimiento. Sin embargo, es posible afirmar que nada de esto significa una cuota de menor compromiso con la empresa y sus funciones, si no, todo lo contrario. El compromiso es distinto al que se tenía en el pasado.

Las palabras recurrentes en las entrevistas a Millennials han sido: resultados versus control, movilidad/home-office versus locación institucional, espacio de trabajo compartido versus box según jerarquía. Valoran la posibilidad que da la tecnología de disponer de sus propios horarios, estando el acceso al trabajo presente en todo lugar y a toda hora. En verdad, pareciera que, para esta generación, es más importante disfrutar el camino que llegar al destino final. Saben reconocer oportunidades para conectar la pasión y sus propósitos con el trabajo y el esfuerzo. Participan de ONGs. Quieren marcar una diferencia en el mundo. El sentido de familia y de matrimonio es otro.

Respecto a la generación $Z$, se observaron características similares a las de cualquier adolescente de su época: tienden a no escuchar a sus mayores, no tienen en cuenta lo que otros tienen que decir y sólo prestan atención a sus pares, los de su edad (esto es igual a los Baby
Boomers en los 50/60). Para ellos prevalece la inteligencia y el conocimiento tecnológico sobre las apariencias físicas, identidad de género o cualquier otro diferenciador. Su "sociedad" existe en internet, donde se abren sus mentes y expresan sus propias opiniones sin tapujos. Pueden estar presencialmente en un grupo y seguir conectados con sus dispositivos móviles con comunidades virtuales. A eso llaman ellos estar. No existe, para ellos, un mundo virtual y un mundo real, sino que existe un sólo mundo. Mostraron ser impacientes, hiper-informados y se precian de influir en las compras familiares porque creen tener más y mejor información que sus padres a la hora de decidir - y así suele ser, efectivamente.

Las entrevistas realizadas han permitido visualizar una gran coincidencia, independientemente de la generación con la que cada uno se identifique, respecto a que el mayor impacto que se recibe en la vida está dado sobre la identidad, los valores y desvalores. Se identificaron cuatro etapas en las que se replantean y re jerarquizan los valores individuales: La primera etapa de formación de identidad se da en el núcleo familiar primario; la segunda en la educación formal; la tercera en los ambientes laborales; y existe una cuarta que se da cuando se ha tenido una expatriación. En este caso, muchos de los entrevistados que pasaron por esa experiencia, manifestaron el haber sentido dificultades de adaptación al regresar a su patria.

En general los entrevistados manifestaron la necesidad de sentirse parte de una identidad dominante. Para muchos de ellos, la cronológica está perdiendo total relevancia, dado que hay conductas que teóricamente deberían tener los individuos de una generación específica, pero que no fueron reconocidas por los entrevistados 
como aporte sino como privativas de una u otra generación. Eran y son percibidas en la mayoría de los casos como un problema y en los mejores casos como desafío.

Finalmente, muchos reconocieron tener características de una generación distinta a la que correspondía por el estereotipo cronológico, pero atender a esas características no aseguraba seguir perteneciendo al mundo económico-productivo. El pertenecer a una generación no aseguraba permanencia en su organización.

Continuando con el análisis de resultados del censo, se procede con la presentación de la información recabada mediante la boleta censal.

La Figura 1 muestra, según la percepción de la población, los factores determinantes para poder adaptarse a las demandas del mercado y a la volatilidad del mundo actual.

\section{Figura 1}

Adaptación a demandas del mercado laboral y al volátil mundo actual

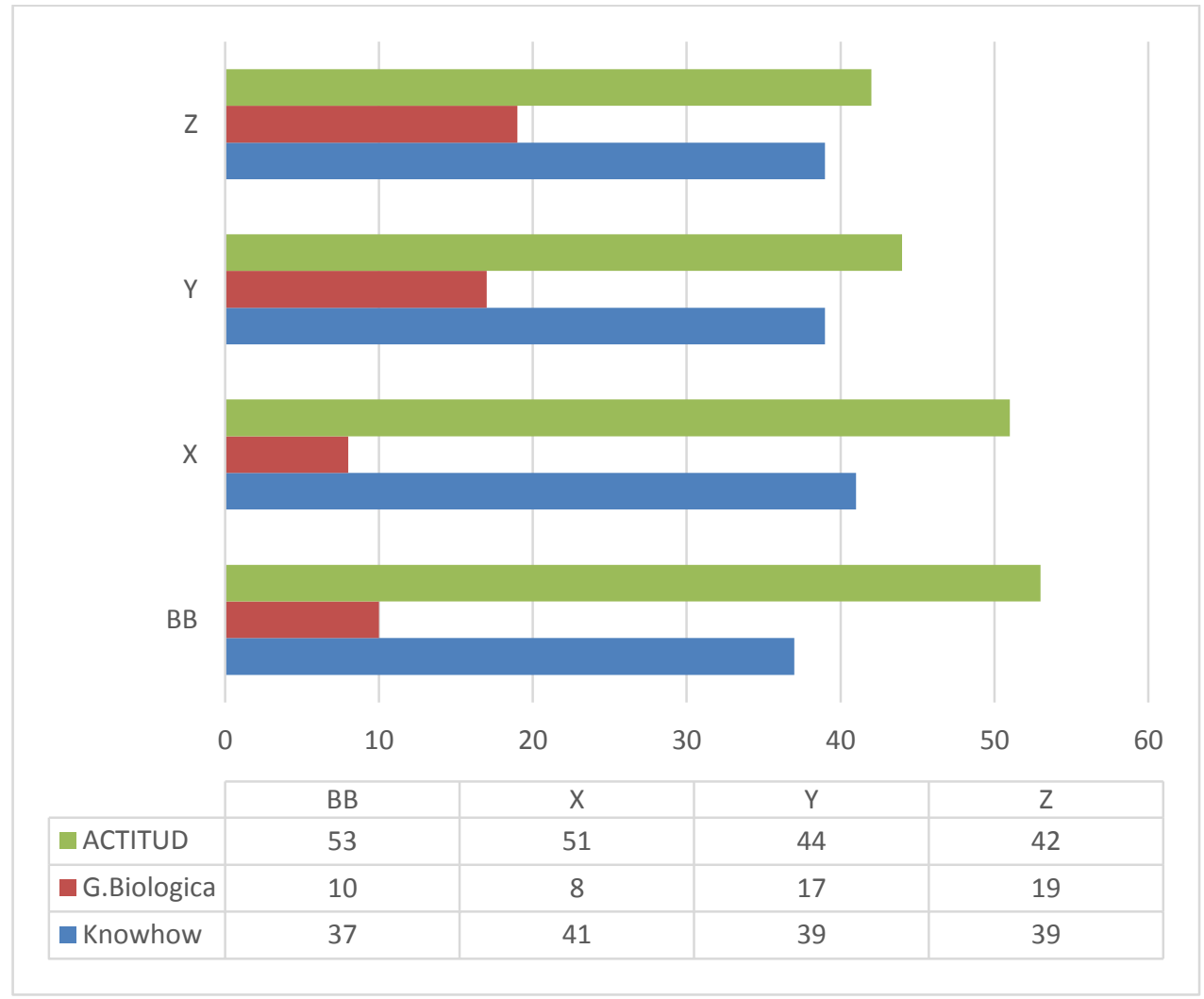

Según lo evidencia la Figura 1, los 4 grupos generacionales consideran a la Actitud como el factor más determinante para poder adaptarse al volátil mundo actual (mayor o igual al 42\%). Según los resultados obtenidos, el segundo factor es el Conocimiento o Know-how (mayor o igual al 37\%). El factor Generaciones es considerado determinante para menos del 20\% de la población. Asimismo, la Figura 2 dio a conocer la percepción del grado en que contribuye positivamente cada uno de los criterios establecidos ante el desafío de una innovación y la necesidad de aceptarla y alinearse a ella en el mundo laboral. 


\section{Figura 2}

Determinantes del logro de desafíos de innovación

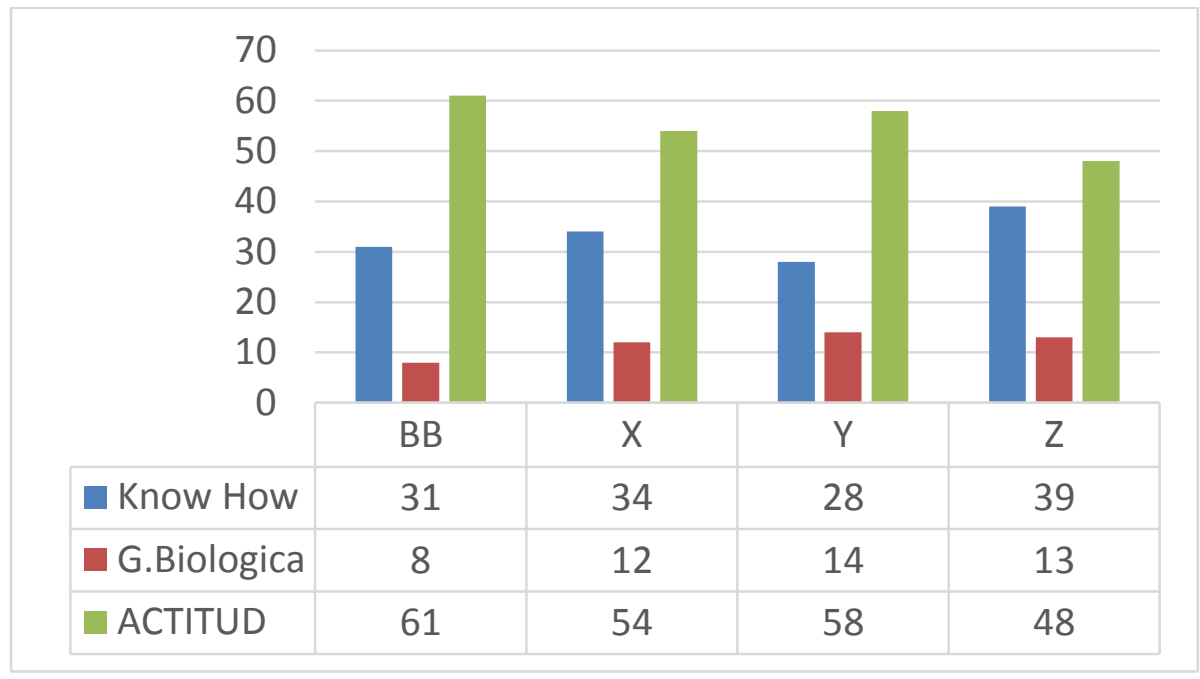

En la Figura 2 se muestra que la población afirma que el mayor factor determinante para lograr desafíos de innovación es la Actitud; su impacto como factor crítico supera el $48 \%$; asimismo, entre el 28 y 39\%, dependiendo de la generación, identifican como principal factor el Conocimiento; por su parte, entre el 8 y $14 \%$ consideran que el principal factor es el generacional.

Sucede algo similar cuando se solicita priorizar los 3 factores en términos de importancia en el éxito de su carrera profesional, tal como se evidencia en la Figura 3.

\section{Figura 3}

Determinantes del éxito en la carrera profesional

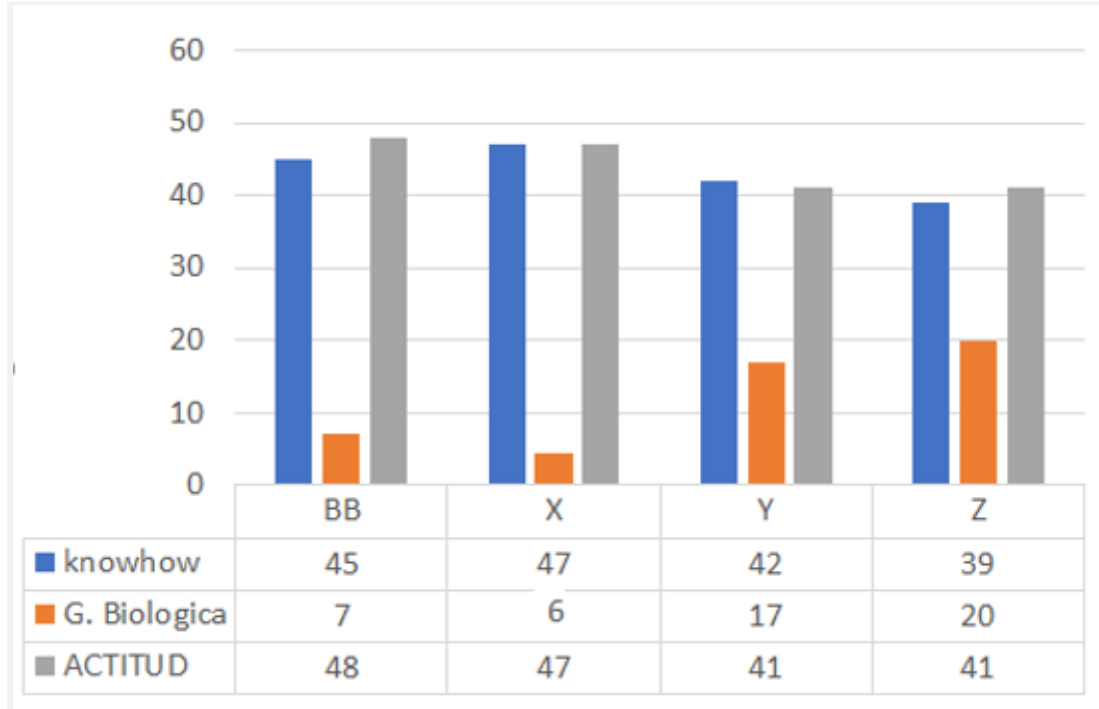


Se ve en las figuras 1,2 y 3 que en cada pregunta se cumple el mismo patrón de respuesta; es decir, la Actitud es siempre el principal factor reconocido como crítico positivo para ser exitoso. Se confirma en la
Figura 4, donde las 4 generaciones coinciden en que la Actitud es el principal factor determinante para solucionar situaciones de conflicto.

\section{Figura 4}

Criterios que contribuyen a solucionar una situación de conflicto

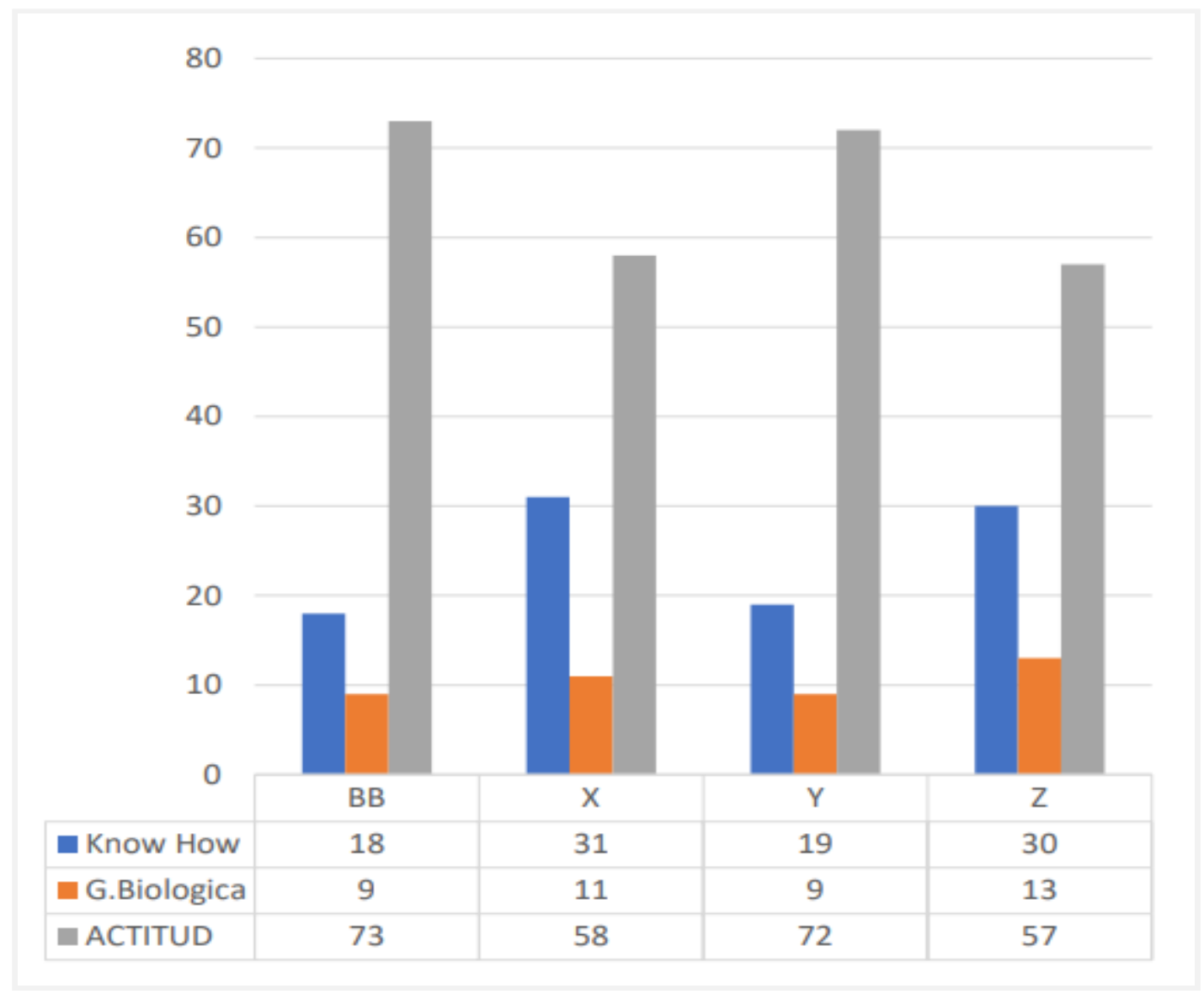

En la Figura 4 se observa que el mayor factor determinante para lograr solucionar situaciones de conflicto en el trabajo es la Actitud; su impacto como factor crítico supera el 57\%; asimismo, entre el 18 y 31\%, dependiendo de la generación, identifican como principal factor el Conocimiento; por su parte, entre el 9 y $13 \%$ dan prioridad a la generación a la que se pertenece. 


\section{Figura 5}

Criterios que contribuyen a abordar un cambio de tecnología en el ámbito laboral

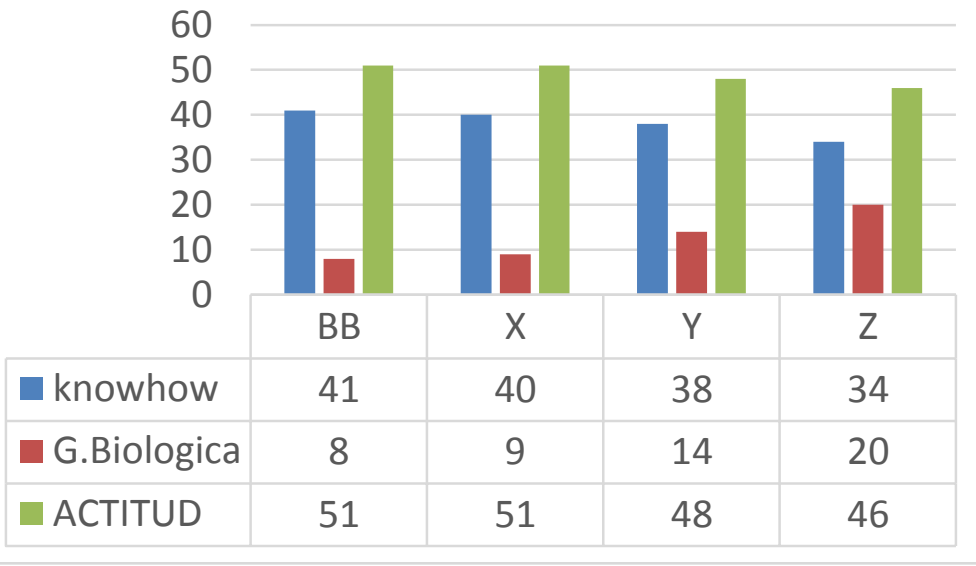

Los resultados presentados en la Figura 5 muestran que existe una percepción similar en los grupos generacionales respecto al abordaje de adelantos tecnológicos; la Actitud y el Conocimiento superan al factor generacional.

\section{Figura 6}

Criterios que favorecen su conducta de fomento de la Responsabilidad Social Empresarial

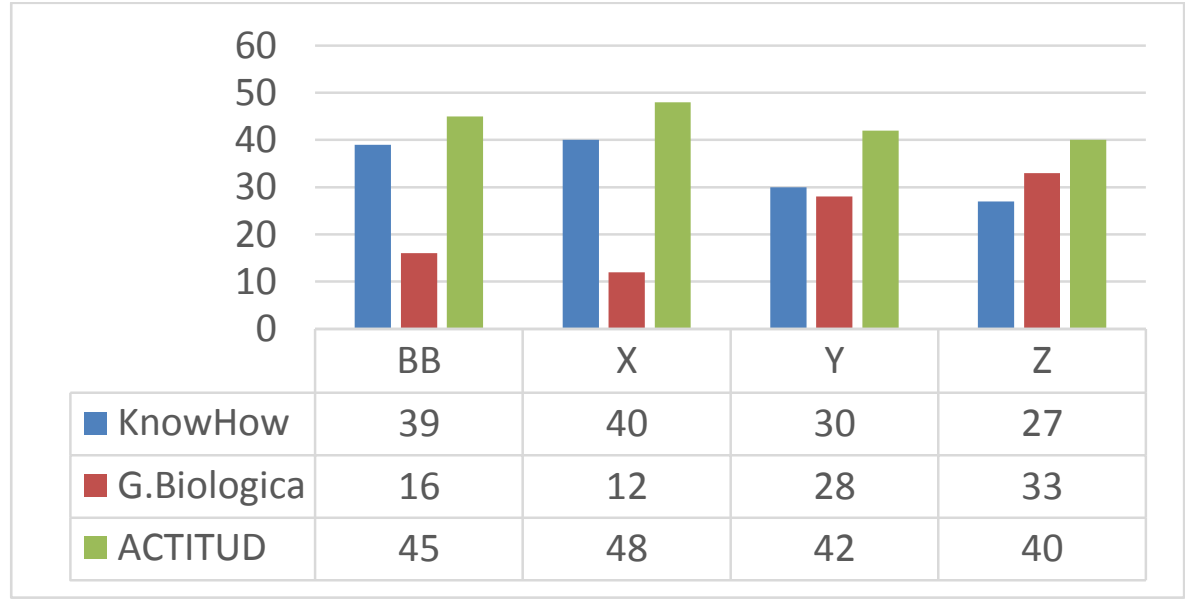

Se analizaron también los factores que favorecen una conducta de fomento de responsabilidad social empresarial, los resultados se presentan en la Figura 6 . En este caso resalta la percepción de la generación $\mathrm{Z}$ que considera la Actitud, la Generación y el Conocimiento muy similares en importancia; asignando 40\%, 33\% y $27 \%$ respectivamente.
Continuando con las observaciones, si bien los códigos de cada generación son específicos y condicionan la forma de mirar la realidad, esto sucede sólo en contextos determinados y no en todos; paralelamente, las diferencias de perspectivas generacionales -demográficas- han sido y son históricamente permanentes. No es "novedad" la disparidad de visiones de cada 
generación. Eso ha sido comprobado a lo largo de décadas y siglos. Si se analiza la gran problemática de las empresas familiares, se ve que esta diversidad generacional es la que ha causado la mayoría de las crisis en ese tipo de organización, por lo menos desde el 1920 en adelante; tanto que generó una Teoría de Gestión propia con el fin de resolver específicamente dichos problemas. Claramente los códigos de cada generación son distintos, no así el conflicto emanado de las perspectivas diferentes, que muchas veces son hasta críticamente contradictorias. A modo de ejemplo sive nombrar a empresas como Ford y su historia critica familiar.

\section{DISCUSIÓN}

Se observa que muchas investigaciones realizadas en otras geografías y culturas tales como: El concepto de generación en las teorías sobre la juventud de Leccardi y Feixa en Chile (2011); o Los millennials y la investigación de generaciones, de Esomar's Research World Magazine en Estados Unidos (2012); revelan que madurar en un mundo de transformación acelerada deja en las generaciones $Y$ y $Z$ un sentimiento de inquietud respecto al futuro.

Cuando se abordan estos casos, por ejemplo, para comparar los resultados de esta investigación, se observa que solo existen matices de diferencias que están dados por pautas culturales y entornos. Estas diferencias se diluyen cuando la vida transcurre en grandes ciudades integradas a la globalidad del mundo de hoy. Sin embargo, "es importante esta perspectiva para no tentarse y caer en generalizaciones que empañarían cualquier análisis del proceso de investigación" (Hofstede, 2001).
Al entender la dinámica, tanto en instituciones, organizaciones, comunidades e individuos, se podrá decidir conscientemente si se quiere pertenecer sólo a una generación que divida, o a una unificadora que ubique a los individuos en el centro del mundo actual; que los identifique con la adaptabilidad, con el cambio, con la capacidad de ser un actor relevante en el nuevo orden. Una es por biología y/o cronología y la otra, la generación " $M$ ", es por elección. Una apela al determinismo y la otra a la libertad del individuo.

Sólo hace falta recordar que siempre hubo una generación de jóvenes que sorprendía a sus mayores por sus radicales cambios en sus perspectivas. Desde los Hippies a los Punks, la realidad parcial siempre contrastó contra la supuesta realidad establecida (Romaní \& Sepúlveda, 2018). Un rasgo que se distingue como patrón común es que existe un punto de tensión entre las visiones que se tengan del presente y futuro, independientemente de a que generación se pertenezca.

También, que los valores que anidan en la memoria, tanto como los nuevos que pujan por su supremacía, están siempre listos para influir en las conductas de los interrogados. Son dos génesis que delimitan las necesidades constantes de superación del individuo.

¿Y por qué los entrevistados ven esto? porque la celeridad de los cambios es tan vertiginosa, las verdades son tan efímeras y ambiguas, la vida de las empresas es tan frágil y compleja que la única verdad es la mutación. En este contexto, por lo tanto, una actitud desafiante sobre uno mismo y los valores puede dar como resultado la adaptación. La Neurociencia impone como competencia clave la flexibilidad cognitiva que no es ni más ni menos que la capacidad de 
aceptar los cambios con resiliencia. Tomándolos como un hecho.

Alvin Toffler (1971), experto en administración, en su libro "el Shock del Futuro" ya habla de los analfabetos del Siglo XXI, como aquellos que no tienen la actitud de aprender, desaprender y reaprender." Estas 3 acciones son condiciones esenciales para una mutación positiva. Toda persona deviene en una subjetividad que requiere entenderse, en términos de quién es en distintos momentos de su vida. Para la población, la observación no se puede agotar en un solo momento ni por un único "accidente" como la fecha de nacimiento, ya que su "objetividad" consiste, justamente, en comprender la diversidad que supera su identidad.

Lo difícil, según expresó la población, es la aceptación de esa realidad que se traduce en la propensión, o no, al cambio. En tener la vitalidad de continuar o de rendirse ante esas realidades, dadas por las relaciones múltiples $y$ heterogéneas que se presentan en el uso de la tecnología y en las relaciones de interdependencia recíproca con otros. Existe un fuerte sentimiento a permanecer $y$ de no ser etiquetados; a desafiarse y a superarse uno mismo. Cuando piensan en crecer y desarrollarse, lo último que se acuerdan es de la generación a la que pertenecen y en cambio, lo primero que saben es que deben aceptar adaptarse $y$ "aggiornarse" / actualizarse; y en lo posible, anticiparse a los desafíos que el contexto impone.

El paradigma tradicional de clasificación de las generaciones ha muerto. Un nuevo paradigma inclusivo nace y este es, el que los autores de este artículo científico han denominado la generación " $\mathrm{M}$ ", de la mutación y metamorfosis permanente. El que sobrevivirá no será por su edad cualquiera sea ésta, sino por su capacidad de adaptarse positivamente a este mundo disruptivo. Cada uno de los seres humanos se constituye en veedor y desarrollador de la generación $\mathrm{M}$. Esta es una generación en la que se decide consciente el pertenecer o no.

La pertenencia superadora es y será la generación $M$, o si no lo individuos se quedan aislados en su propia burbuja, no modificable. La hiperinformación, conexión constante e inmediatez es una característica de la cotidianeidad y no puede decirse que impacte en una generación y no en otra. Tiende a abrazar a unas más rápido que otras, pero todas la necesitan con igual criticidad.

Una gran mayoría de los consultados sintieron que forman parte de: Comunidades en línea como LinkedIn, Orkut, Google+, Flickr, Twitter, Tuenti, Facebook, Glassdoor, Indeed, y tantas otras. Esto permite a las personas conectar con comunidades multitudinarias y acceder a extraordinarios aportes intelectuales. Todo sucede sin conocer personalmente más que a un mínimo porcentaje, en forma personal y profunda. El saber es abordado con múltiples formas de lectura transmedia. La Neurociencia ya admite que se está generando un tipo de sinapsis nuevo en el cerebro, sin saber aún el impacto que tendrá (las pantallas táctiles parecieran ser una de las causas). Estas narrativas tienen una nueva "lógica, la digital". Y esto le sucede a cualquiera que esté conectado y no sólo a los de una generación.

Se infiere, entonces, que se trata de un cambio de lógicas en la mente (cambio de comportamiento) de la población estudiada. El modo de definir las relaciones ha dejado de ser binario y privativo de una generación. Este es el mundo de la incertidumbre constante. Si antes el género era masculino o femenino; hoy, la 
homosexualidad ha dejado de ser la única alternativa a la heterosexualidad. Son más de cincuenta las identidades de género y también son independientes a la edad o grupo etario.

La nueva economía se muestra disruptiva, en una abundancia aún incomprendida, y demandante de nuevas profesiones. He aquí la actitud clave requerida, para reinventar las profesiones y estilo de vida una y otra vez, optimista y sin fatiga. Paul Irving (2018) expone una "estrategia de longevidad" para fomentar una fuerza laboral multigeneracional vibrante. El objetivo de las personas por encontrar armonía en sus vidas ha, finalmente, puesto en escucha activa a las organizaciones y las han llevado a la situación de que cuestionen sus propias estructuras, sus formas de trabajar y los ambientes de trabajo. También está en tela de juicio lo que se entiende por compromiso, lealtad, contracción al trabajo, libertad, disciplina, control, liderazgo, etc.

Esto implica un reto insoslayable y hasta traumático. Se entiende que la estrategia empresarial exige atraer al talento adecuado (Aquino et al., 2010). Paralelamente, necesitan que permanezcan los profesionales capaces de actualizar constantemente su idoneidad. Asimismo, que dejen un legado superador, para las generaciones venideras. La realidad dinámica, versátil, incierta y compleja nos invita a participar voluntariamente de la generación $M \circ$ de mutación / adaptación positiva permanente.

El panorama es claro, ya existe otra forma de entender el éxito, otra forma de resolver los problemas. Según Peter Drucker (2013) las organizaciones que permanezcan serán las que más rápidamente cambien su forma de hacer las cosas. Es en esas organizaciones donde el proceso social tendrá las consecuencias económicas que les permitirá: sobrevivir, no quedar rezagadas o extinguirse.

Es importante señalar que existen aspectos clave aun para investigar a fin de poder decir que los resultados son aplicables a una perspectiva mundial. Se debe aún explorar representantes sin formación académica y con formación académica -hasta carrera universitaria de grado- para las mismas 4 generaciones, objeto de este trabajo. También queda por abordar otros países de Latinoamérica, Norteamérica, Europa, países asiáticos que marcan tendencia. Paralelamente, también explorar otras generaciones como la "Alpha" cuando estos lleguen al mercado laboral.

Otro aspecto relevante para investigar desde la Ciencia Política sería ir más allá del mundo laboral. Este es un comienzo esencial que contradice una verdad que parecía irrefutable, los resultados en todo caso corroboran la existencia unificadora de la generación " $\mathrm{M}$ ". Lo que prevalece es la forma de ayudar a alguien a aprender y cambiar (Boyatzis et al., 2019).

\section{CONCLUSIONES}

Las generaciones normalmente estereotipadas tienen la misma impronta particular que tuvo cada nueva generación anterior al insertarse al mundo: una nueva perspectiva, una nueva mirada, unos nuevos objetivos, ni más ni menos importantes y desestabilizadores del equilibrio imperante hasta ese momento. Se debe hablar entonces de la existencia transversal de una generación que los autores de este artículo denominan "generación Metamorfosis" o generación " $M$ ", y que es común a cualquiera de las categorías/generaciones investigadas. Esta generación $\mathrm{M}$ es y será factor común para todo el que quiera pertenecer al Sistema (empresario- 
profesional-trabajador) y no determinado por algún rango etario en especial, sino por la necesidad de adaptarse a las imposiciones del contexto VICA, de las Transformaciones Tecnológicas y de las permanentes disrupciones; tanto desde el punto de vista del negocio como de los hábitos cambiantes (de los usuarios y clientes). Dicha generación es supra las generaciones e independiente de ellas, con la que cada uno se identifique, ya que el mayor impacto que se recibe en la vida profesional laboral no está dado ni por el rango etario, ni valores y desvalores, ni por la identidad de género.

Dos son las directrices que llevan al punto de confluencia para tener la capacidad de permanecer en el mercado laboral: Primero, el manejo de las nuevas tecnologías, cualesquiera sean ellas; segundo, nuevos valores y actitudes como flexibilidad cognitiva y diversidad, respectivamente, desaprender, reaprender $y$ aprender.

El resultado concluido a partir del trabajo de investigación realizado ha sido muy distinto, se ha notado una evidencia paradigmática drásticamente distinta de la postulada por los que ponen toda la energía en el impacto de las generaciones, como si eso hubiese sido disruptivo de comportamientos históricos. Se considera prudente resaltar el enfoque actitudinal de los consultados, que supera las particularidades que de alguna manera anularían la impronta individual y el libre albedrio. Este abordaje, desde la clasificación simplista y casi superflua de lo etario o cronología generacional, puede dar un código común pero no una única razón para sus comportamientos; están las cuatro generaciones en un mismo punto de partida o en un mismo barco en aguas turbulentas, que es el hoy y lo que determinará la misión de rescate será: primero, la actitud de cada uno y como grupo; en segundo lugar, el conocimiento aportado; y recién como último factor estará su pertenencia a un grupo etario. Se trata de un cambio de paradigma, de tornar de una perspectiva de escasez a una de abundancia, de una de conflicto generacional a otra de identidad "multiedad"; en un contexto de complejidad, incertidumbre, ambigüedad y vulnerabilidad, de un viejo orden a un nuevo orden mundial, de statu quo donde unos predominan sobre otros a la disrupción, contradicción y hasta oposición en un nuevo mundo político, social, económico y del trabajo.

\section{REFERENCIAS}

Aquino, J; Vola-Luhrs, R; Arecco M \& Aquino, G. (2010). El Rol de los Recursos Humanos y la empresa. 1ra. Edición en Prentice Hall Pearson Educación. Capítulo 2, pág. 33. ISBN 978-987-615-097-2. CDD 658.3. Buenos Aires

Boyatzis, R; Melvin Smith, Ellen Van Oosten Helping People Change. (2019) Libro / Artículo de, Harvard Business Review. Julio 2019

https://www.google.com/search?q=Boyatzis $\% 2 \mathrm{C}+\mathrm{R} \% 3 \mathrm{~B}+$ Melvin+Smith\%2C+Ellen+Van +Oosten+Helping+People+Change.+Libro+ $\% 2 \mathrm{~F}+\mathrm{Art} \% \mathrm{C} 3 \%$ ADculo + de\%2C + Harvard +Bu siness+Review.+Julio $+2019 \&$ oq $=$ Boyatzis $\% 2$ $\mathrm{C}+\mathrm{R} \% 3 \mathrm{~B}+$ Melvin +Smith\%2C+Ellen+Van+O osten+Helping +People+Change. + Libro+\%2 $\mathrm{F}+$ Art\%C3\%ADculo+de\%2C+Harvard+Busin ess+Review. +Julio +2019+\&aqs =chrome..69i 57.817j0j15\&sourceid $=$ chrome\&ie $=$ UTF-8

Concejo Edurne. (2018). ¿Cómo eres? Descubre a que generación perteneces, según tu fecha de nacimiento. La Vanguardia. Barcelona 15.7.18 Taxonomía de generaciones. Pág 2 https://www.lavanguardia.com/vivo/2018040 8/442342457884/descubre-que-generacionperteneces.html 
Corica, J. \& Dinerstein P. (2009). Diseño curricular y nuevas generaciones: incorporando a la generación Net. 1ra. Ed. Mendoza: Editorial Virtual Argentina

Drucker, P. F. (2013). La sociedad poscapitalista. Sudamericana.

Esomar's Research World Magazine. (2012). Los millennials y la investigación de generaciones. International Research, Esomar's Research World Magazine. Estados Unidos

Espiritusanto, O. (2016). Los auténticos nativos digitales: ¿Estamos preparados para la Generación Z? Revista de Estudios de Juventud. Número 114. Diciembre 2016. http://www.injuve.es/ca/node/70673

Gagneten, A. M.; Imhof, A.; Marini, M. D. R.; Tomas, P.; Amavet, P.; Ravera, L. \& Ojea, N. (2008). Biología: conceptos básicos. Universidad Nacional del Litoral

Hofstede, Geert. (2001). Culture's Consequences, Amazon. Second Edition. https://www.hofstedeinsights.com/product/compare-countries/

Irving, P. (2018). The Aging Workforce, your workforce will keep older. ¿Do you have a Strategy? Harvard Business Review. November

https://www.google.com/search?q=Irving\%2 $\mathrm{C}+$ Paul+The+Aging+Workforce $\% 2 \mathrm{C}+$ your+ workforce+will+keep+older.+\%C2\%BFDo+y ou+have+a+Strategy\%3F+Harvard+Busines $\mathrm{s}+$ Review. + November $+2018 \&$ oq =Irving\%2C + Paul+The+Aging +Workforce\%2C\&aqs $=$ ch rome.2.69i57j69i59l3.8108j0j7\&sourceid $=\mathrm{chr}$ ome\&ie $=$ UTF- 8

Leccardi, C., \& Feixa, C. (2011). El concepto de generación en las teorías sobre la juventud. Última década, 19(34), 11-32.

Pinnel, A.P. \& Vola-Luhrs, R. (2020). Anticuerpos - Secuelas Positivas. ¿qué aportará el COVID19 a las Organizaciones? News Letter Voyer International pág.1

Romaní, O \& Sepúlveda, M. (2018). Estilos juveniles, contracultura y política. Styles juvéniles, contre-culture et politique. Youth styles, counterculture and politics. Revista Latinoamericana.

https://journals.openedition.org/polis/5769

Toffler, A. (1971). Future Shok en Iberlibro.com 1971 ISBN 10: 0553101501 - ISBN 13: 9780553101508

Uviz, D.S. (2008). La gestión del talento en la era del conocimiento. Tesis. Universidad De Buenos Aires Facultad de Ciencias Económicas. Carrera de Posgrado de Especialización y Gestión Estratégica de Recursos Humanos

Vola-Luhrs, R. (2007). Recursos humanos: lo que sobra es el talento. - 1a ed. Pág. 27- Buenos Aires: Universidad Libros, 2007.ISBN 978987-9465-60-8. 1. Recursos Humanos. I. Título. CDD 658.3 